\title{
Erratum to: Acromegaly: surgical results in 548 patients
}

\author{
Cecilia Fernández Mateos ${ }^{1} \cdot$ Maria García-Uria $^{2}$ Tomás Lucas Morante ${ }^{3}$. \\ José García-Uria ${ }^{1}$
}

Published online: 5 July 2017

(C) Springer Science+Business Media, LLC 2017

\section{Erratum to: Pituitary DOI 10.1007/s11102-017-0813-y}

The original version of this article unfortunately contained a mistake in text and table header. The corrections are given below. The original article has been corrected.

In Preoperative hormonal values section, the PRL must be $>100 \mathrm{ng} / \mathrm{ml}$, not $>00 \mathrm{ng} / \mathrm{ml}$ as published. The correct paragraph is given below:
PRL evaluation was realized in 453 patients (82.6\%). Hyperprolactinemia $>5 \mathrm{ng} / \mathrm{ml}$ was associated with acromegaly in 95 patients (20.9\%), (64 women and 31 men.). PRL $>100 \mathrm{ng} / \mathrm{ml}$ was found in twelve patients $(2.64 \%),(7$ women and 5 men).

The column headers of Table 3 are not in order. The corrected Table 3 is given below:

The online version of the original article can be found under doi:10.1007/s11102-017-0813-y.

Cecilia Fernández Mateos

valdelagua@hotmail.com

Maria García-Uria

mariagarciauria@hotmail.com

Tomás Lucas Morante

tlucasmorante@gmail.com

José García-Uria

neuromic@telefonica.net

1 Neurosurgery Department, Puerta de Hierro Hospital, Madrid, Spain

2 Emergency Medicine Department, Puerta de Hierro Hospital, Madrid, Spain

3 Endocrinology Department, Puerta de Hierro Hospital, Madrid, Spain 
Table 3 Postoperative

GH-levels (548 patients)

\begin{tabular}{lllll}
\hline Period & GH $>5 \mathrm{ng} / \mathrm{ml}$ & GH 5-2.5 ng/ml & GH 2.4-2 ng/ml & GH $<2 \mathrm{ng} / \mathrm{ml}$ \\
\hline $1975-84$ & $40(51 \%)$ & $7(9 \%)$ & $2(3 \%)$ & $29(37 \%)$ \\
$1985-94$ & $39(25 \%)$ & $9(6 \%)$ & $6(4 \%)$ & $101(65 \%)$ \\
$1995-04$ & $35(17 \%)$ & $11(5.4 \%)$ & $11(5.4 \%)$ & $148(72.2 \%)$ \\
$2005-15$ & $19(17 \%)$ & $7(6 \%)$ & $6(5 \%)$ & $78(70 \%)$ \\
Total patients & $133(24.2 \%)$ & $34(6.2 \%)$ & $25(4.5 \%)$ & $356(65 \%)$ \\
\hline
\end{tabular}

*Surgical results (1975-2015) Postoperative GH 2-21 days after surgery 